\title{
Interference Effects on Double Quantum Dots Coupled Between Metallic and Superconducting Leads
}

\author{
J. BARAŃski AND T. DOMAŃSKi \\ Institute of Physics, M. Curie Skłodowska University, 20-031 Lublin, Poland
}

\begin{abstract}
We describe the quantum interference effects in the nanodevice consisting of the double quantum dot coupled to the metallic and superconducting electrodes. In such heterostructures the superconducting properties are spread to the quantum dot due to the proximity effect. We investigate the density of states and anomalous Andreev conductance of the interfacial quantum dot exploring the conditions necessary for appearance of the Fano-type lineshapes. We also consider the electron correlations and discuss an interplay between the Coulomb blockade and the Fano-type interference.
\end{abstract}

PACS: 73.63.Kv, 73.23.Hk, 74.45. $+\mathrm{c}, 74.50 .+\mathrm{r}$

\section{Introduction}

When nanoobjects like quantum dots (QDs) are connected to superconductor (S) the Cooper pairs may diffuse from $\mathrm{S}$ to QD [1]. The diffusion is possible because the quantum dot behaves like a superconducting grain [25]. This phenomenon is known as the proximity effect.

With such quantum dot coupled to superconducting and metallic leads the indirect Andreev current via quantum dot becomes possible. In fact, in the extreme limit $\Delta_{p} \rightarrow \infty[2,4]$ the single particle current is forbidden and the Andreev reflection is the only possible transport channel.

Heterostructures involving the quantum dots coupled to superconducting and metallic leads represent also the attractive field to investigate an interplay between the superconducting order and correlation effects [1]. Usually the on-dot pairing is strongly suppressed by the Coulomb repulsion but, if QD is coupled to superconductor with the strength $\Gamma_{S}$ much bigger than the coupling $\Gamma_{N}$ to the normal metal, the proximity induced pairing could be dominant. Here we focus on the regimes where such correlation effects and the superconducting order can coexist.

Some aspects of a competition between the correlations and proximity effect have been so far addressed by a number of authors (see e.g. the review paper [6] and other references cited therein) using various techniques ranging from the perturbative expansions, the auxiliary fields to the exact solution within the numerical renormalization group scheme. Besides the rather obvious effect of the Coulomb blockade it has been also argued [6] and later on confirmed experimentally [1] that for $\Gamma_{S} \approx \Gamma_{N}$ the formation of the Kondo resonance slightly enhances the subgap Andreev conductance.

In the present study we discuss the interference effects that occur in a presence of additional degrees of freedom. For this purpose we consider the side-coupled quantum dot which is connected only to the interfacial QD (Fig. 1). The additional quantum dot allows for an extra pathway when electrons have the energies $\epsilon_{2}$, corresponding to the levels at the side-coupled quantum dot [7].

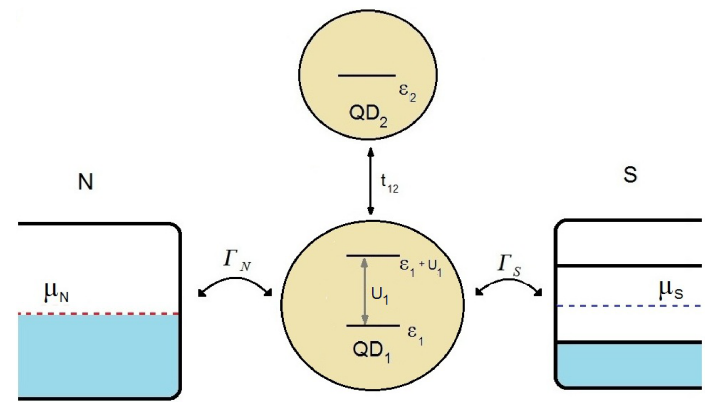

Fig. 1. Schematic illustration of the double quantum dot coupled in the T-shape configuration to the metallic $(\mathrm{N})$ and superconducting (S) leads.

\section{The model and method}

We start with the Anderson impurity Hamiltonian

$$
\hat{H}=\hat{H}_{N}+\hat{H}_{S}+\hat{H}_{D Q D}+\hat{H}_{t},
$$

where

$$
\begin{aligned}
& \hat{H}_{N}=\sum_{k, \sigma} \xi_{k} \hat{C}_{k, \sigma}^{\dagger} \hat{C}_{k, \sigma}, \\
& \hat{H}_{S}=\sum_{p, \sigma} \xi_{p} \hat{C}_{p, \sigma}^{\dagger} \hat{C}_{p, \sigma}-\sum_{p}\left(\Delta_{p} \hat{C}_{p, \uparrow} \hat{C}_{-p, \downarrow}+\text { h.c. }\right),
\end{aligned}
$$

are respectively the Hamiltonians of metallic/superconducting reservoirs with the energies 
$\xi_{k}=\epsilon_{k}-\mu_{\beta}$ measured from the chemical potentials $\mu_{N}$ and $\mu_{S} . \hat{C}_{k, \sigma}^{\dagger}\left(\hat{C}_{k, \sigma}\right)$ are creation (annihilation) operators in metal (index $k$ ) and superconductor (index $p$ ) in two possible configurations $\sigma=\uparrow, \downarrow$ and $\Delta_{p}$ is the energy gap of superconductor. The double quantum dot (DQD) nanostructure is described by the Hamiltonian

$$
\begin{aligned}
& \hat{H}_{D Q D}=\sum_{\sigma, i} \epsilon_{i} \hat{d}_{i, \sigma}^{\dagger} \hat{d}_{i, \sigma}+t \sum_{\sigma}\left(\hat{d}_{1, \sigma}^{\dagger} \hat{d}_{2, \sigma}+\text { h.c. }\right) \\
& \quad+U_{1} \hat{n}_{1, \uparrow} \hat{n}_{1, \downarrow},
\end{aligned}
$$

where $\epsilon_{i}$ is energy of each quantum dot (interfacial $i=1$ and orbital $i=2$ ), $t$ denotes the hopping integral between the quantum dots and $U_{1}$ is the on-dot Coulomb repulsion. As usually, $\hat{d}_{i, \sigma}^{\dagger}\left(\hat{d}_{i, \sigma}\right)$ stand for the creation (annihilation) operator of $\sigma$ spin electron on the $i$-th dot. Transport phenomena of the setup (Fig. 1) are provided by the hybridization of the interfacial QD to the external electrodes

$$
\hat{H}_{t}=\sum_{k, \beta, \sigma} V_{k, \beta}\left(\hat{d}_{1, \sigma}^{\dagger} \hat{C}_{k, \beta}+\text { h.c. }\right) .
$$

It is useful to introduce the constant coupling strength between the interfacial dot and both reservoirs

$$
\Gamma_{\beta}=2 \pi \sum_{k, \beta}\left|V_{k, \beta}\right|^{2} \delta\left(\omega-\xi_{k}\right),
$$

and we shall use $\Gamma_{N}$ as the unit for energies. To find the density of states and the effective transmittance we need to calculate the matrix elements of the retarded Green's function $G_{1}(\tau)=-T_{\tau}\left\langle\Psi(\tau) \Psi^{\dagger}\right\rangle$ in the Nambu representation $\Psi^{\dagger} \equiv\left(\hat{d}_{1 \uparrow}^{\dagger}, \hat{d}_{1 \downarrow}\right), \Psi \equiv\left(\Psi^{\dagger}\right)^{\dagger}$.

The Dyson equation

$$
G_{1}(\omega)^{-1}=\left(\begin{array}{cc}
\omega-\epsilon_{1} & 0 \\
0 & \omega+\epsilon_{1}
\end{array}\right)-\Sigma_{d_{1}}^{0}-\Sigma_{d_{1}}^{U}(\omega),
$$

consists of the selfefenergy, where $\Sigma_{d_{1}}^{0}$ originates from the non-interacting case $(U=0)$ and $\Sigma_{d_{1}}^{U}$ corresponds to the correlation effects. By focusing first on the noninteracting case we analyze the deep subgap regime $|\omega| \ll$ $\left|\Delta_{p}\right|$, where the selfenergy is given by $\Sigma_{d_{1}}^{0}[9]$

$$
\Sigma_{d_{1}}^{0}=\left(\begin{array}{cc}
-\frac{\mathrm{i} \Gamma_{N}}{2}+\frac{t^{2}}{\omega-\epsilon_{2}} & -\frac{\Gamma_{S}}{2} \\
-\frac{\Gamma_{S}}{2} & -\frac{\mathrm{i} \Gamma_{N}}{2}+\frac{t^{2}}{\omega+\epsilon_{2}}
\end{array}\right) .
$$

\section{Coexistence of the proximity and Fano effects}

Fixing the initial energy of the interfacial quantum dot at zero $\epsilon_{1}=0$ we noticed that energy spectrum $\rho(\omega)=-\frac{1}{\pi} \Im G_{1,11}\left(\omega+\mathrm{i} 0^{\dagger}\right)$ of $Q D_{1}$ is symmetrically split by the induced energy gap $\Delta_{d}=\frac{1}{2} \Gamma_{S}$ (see the solid line in Fig. 2). For $\epsilon_{1} \neq 0$ the quasiparticle peaks move to the energies $\omega= \pm E_{1}= \pm \sqrt{\epsilon_{1}^{2}+\left(\frac{\Gamma_{S}}{2}\right)^{2}}$ and they are weighted by the BCS coefficients $u^{2}, v^{2}=\frac{1}{2}\left(1 \pm \frac{\epsilon_{1}}{E_{1}}\right)$.

The Fano-type resonance/antiresonance lineshapes appear near the energies $\pm \epsilon_{2}$. These shapes are clearly the result of an additional degree of freedom for electrons that can hop in/from the side-coupled QD. For a weak hoping amplitude $t \ll \Gamma_{N}$ the resonance near $\pm \epsilon_{2}$ is due

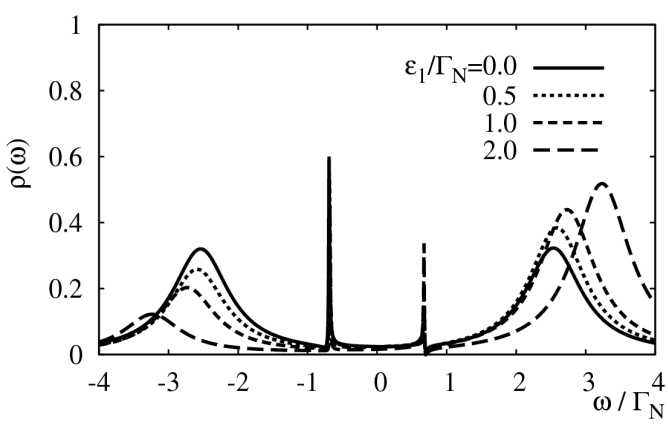

Fig. 2. Density of states of the interfacial quantum dot QD obtained for $\Gamma_{S}=5 \Gamma_{N}, \epsilon_{2}=0.7 \Gamma_{N}, t=$ $0.3 \Gamma_{N}$ and few values of $\epsilon_{1}$. Quasiparticle peaks at $\pm \sqrt{\epsilon_{1}^{2}+\left(\Gamma_{S} / 2\right)^{2}}$ are weighted by the BCS coefficients $\left(u^{2}, v^{2}\right)$ and the interference structures appear around $\pm \epsilon_{2}$

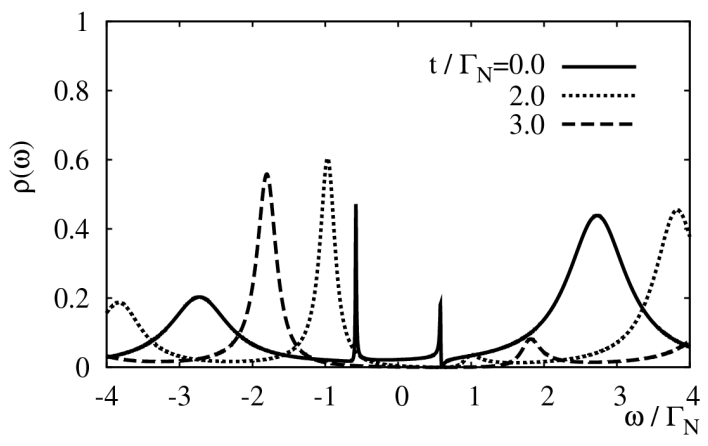

Fig. 3. Density of states of the interfacial quantum dot QD obtained for $\Gamma_{S}=5 \Gamma_{N}, \epsilon_{1}=\Gamma_{N}, \epsilon_{2}=0.6 \Gamma_{N}$ and three values of the interdot hoping parameter $t$. For $t=0.3 \Gamma_{N}$ the Fano lineshapes are formed near $\pm \epsilon_{2}$. For large $t=2 \Gamma_{N}, t=3 \Gamma_{N}$ they evolve into the additional quasiparticle peaks.

to the induced pairing in the side-coupled quantum dot $\left\langle\hat{d}_{2 \uparrow}^{\dagger} \hat{d}_{2 \downarrow}^{\dagger}\right\rangle$. This indirect proximity effect is transmitted via the interfacial QD.

For a relatively small hoping integral $t \ll \Gamma_{N}$ the interference effects are manifested by Fano-type lineshapes formed at energies $\pm \epsilon_{2}$. With increasing parameter $t$ the Fano structures evolve into the new quasiparticle peaks shifted from the initial position $\pm \epsilon_{2}$ (see Fig. 3). This is partly related to an increasing superconducting order of the second quantum dot. The energy level of the sidecoupled QD is significantly split by the induced energy gap.

\section{The Andreev conductance}

Transport properties of our setup may be measured by the differential conductance $\frac{\mathrm{d} I}{\mathrm{~d} V}$. In the regime of $|e V| \ll|\Delta|$ the single particle current is suppressed and the only possible transport occurs via Andreev-type scattering where electron from the normal metal is converted 
to Cooper pair (that propagates in superconductor) with a hole reflection back to the normal metal. We calculate such Andreev current via the interfacial QD using the Landauer-like formula [8]

$$
\begin{aligned}
& I_{A}(V)=\frac{2 e}{h} \int \mathrm{d} \omega T_{A}(\omega)(f(\omega-e V, T) \\
& -f(\omega+e V, T)),
\end{aligned}
$$

where $T_{A}(\omega)=\Gamma_{N}^{2}\left|G_{1,12}(\omega)\right|^{2}$ is the transmittance depending on the off-diagonal parts of the retarded Green's function (7) and $f(\omega, T)$ is the Fermi distribution.

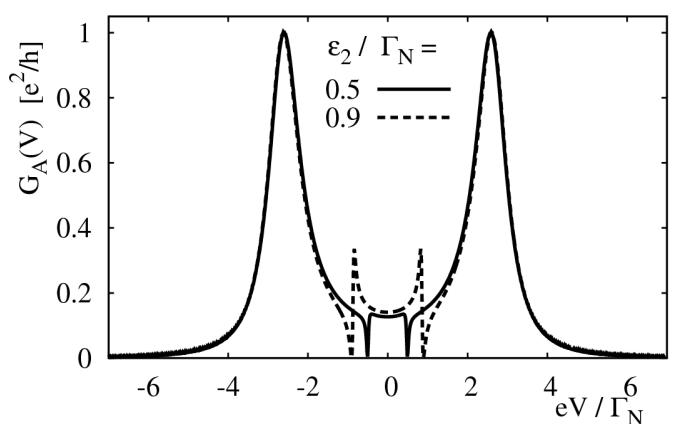

Fig. 4. Differential Andreev conductance $G_{A}(V)=$ $\frac{\mathrm{d} I_{A}(V)}{\mathrm{d} V}$ as a function of the bias voltage $\mathrm{eV}$ obtained for the parameters $\epsilon_{1}=0, \Gamma_{S}=5 \Gamma_{N}, t=0.6 \Gamma_{N}$ and several values of $\epsilon_{2}$. The broad quasiparticle peaks are formed near $\pm \sqrt{\epsilon_{1}^{2}+\left(\Gamma_{S} / 2\right)^{2}}$ and the Fano-type lineshapes are seen near $\pm \epsilon_{2}$.

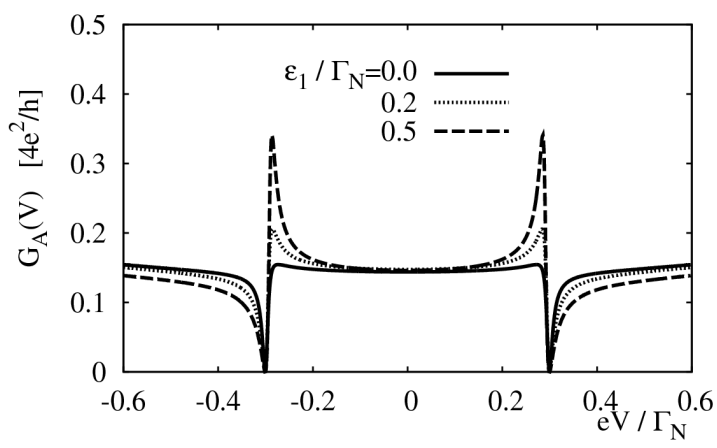

Fig. 5. The Fano-type lineshapes near $\pm \epsilon_{2}$ in the Andreev conductance obtained for $\Gamma_{S}=5 \Gamma_{N}, \epsilon_{2}=0.3 \Gamma_{N}$ and several values of $\epsilon_{1}$.

The indirect proximity effect transmitted onto the sidecoupled quantum dot allows the Cooper pairs to hop between $Q D_{1}$ and $Q D_{2}$. This phenomenon is pronounced in the Andreev differential conductance $G_{A}(V)=\frac{\mathrm{d} I_{A}(V)}{\mathrm{d} V}$. On top of the quasiparticle peaks appearing at $\mathrm{eV}=$ $\pm \sqrt{\epsilon_{1}^{2}+\left(\Gamma_{S} / 2\right)^{2}}$ we notice additional substructures near $\pm \epsilon_{2}$ which take a form of the Fano-type lineshapes as shown in Fig. 4. These Fano-type features are well pronounced for a small hopping integral $t$ and also the energy level $\varepsilon_{1}$ has an influence on their fine structure (see Fig. 5).

\section{Interplay with correlations}

We now inspect the role of Coulomb repulsion $U$ on the density of states in $Q D_{1}$. The main objective of this section is to investigate an interplay between the Fanotype interference with the correlations and the proximity effect.

In order to perceive the correlation effects appearing on the interfacial quantum dot we extend our previous procedure [2] approximating the selfenergy part that corresponds to correlations by the diagonal matrix

$$
\Sigma^{U}(\omega)=\left(\begin{array}{cc}
\Sigma_{N}(\omega) & 0 \\
0 & -\Sigma_{N}^{*}(-\omega)
\end{array}\right) .
$$

By imposing the off-diagonal parts of $\Sigma^{U}(\omega)$ equal to zero we neglect the influence of correlations on the induced on-dot pairing. As long as we stay in the deep subgap regime an eventual suppression of the dot pairing by the Coulomb repulsion seems to be rather justified.

To estimate the selfenergy of our system we use the Hubbard I approximation

$$
\frac{1}{\omega-\epsilon_{1}-\Sigma_{N}(\omega)}=\frac{1-\left\langle n_{1,-\sigma}\right\rangle}{\omega-\epsilon_{1}}+\frac{\left\langle n_{1, \sigma}\right\rangle}{\omega-\epsilon_{1}-U} .
$$

We assume that our system is close to half-filling $\left\langle n_{1 \sigma}\right\rangle \approx$ $1 / 2$ and the energy levels are placed symmetrically with respect to the chemical potential $\epsilon_{1}=-U / 2, \epsilon_{1}+U=$ $U / 2$. With these assumptions we can express the Dyson equation (7) in the following form

$$
G(\omega)^{-1}=\left(\begin{array}{cc}
\frac{\omega^{2}-\left(\frac{U}{2}\right)^{2}}{\omega} & 0 \\
0 & \frac{\omega^{2}-\left(\frac{U}{2}\right)^{2}}{\omega}
\end{array}\right)-\Sigma_{d_{1}}^{0},
$$

where $\Sigma_{d_{1}}^{0}$ is given by the expression (8).

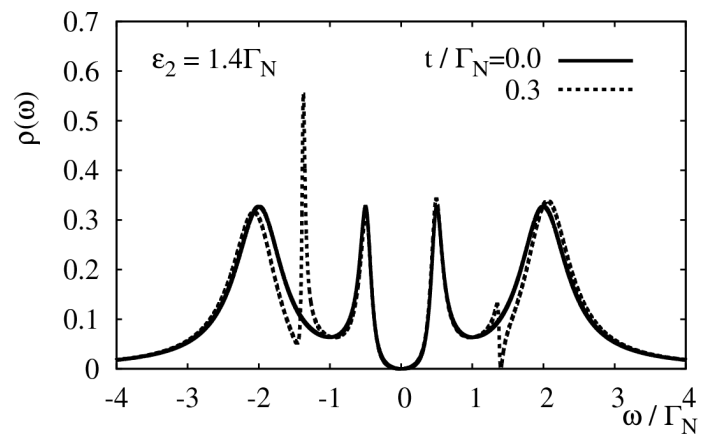

Fig. 6. Density of states of the correlated $Q D_{1}$ obtained in the equilibrium case for $\Gamma_{S}=3 \Gamma_{N}, U=2 \Gamma_{N}$ and $\varepsilon_{2}=1.4 \Gamma_{N}$.

We investigated the influence of quantum interference on the energy spectrum of the correlated quantum dot coupled to superconductor. For the case of the single quantum $\operatorname{dot}(t=0)$ we noticed that in presence of the superconducting electrode both initial energy levels $( \pm U / 2)$ of the interfacial QD are split by an induced energy gap $\Delta_{d}=\Gamma_{S} / 2$. In Fig. 6 we show that additional coupling to the upper quantum dot (with energy $\epsilon_{2}$ ) in- 
duces the Fano superstructures appearing in the energy spectrum near $\omega= \pm \epsilon_{2}$.

\section{Conclusions}

We have analyzed the energy spectrum and charge transport properties of the double quantum dot coupled to the metallic and superconducting leads, focusing on the deep subgap regime $\Delta_{p} \gg|e V|$. We noticed that the superconducting order induced in the interfacial quantum dot may extend onto the side-attached quantum dot which has no direct contact with superconductor. For a weak interdot hoping we observe the Fano-type interference in the spectral function and in the Andreev conductance, while for $t \simeq \Gamma_{N}$ the quantum interference leads to the additional quasiparticle peaks. We have explored the differential conductance in the regime $|e V| \ll \Delta$, where electron transport is possible only through the Andreev reflection. We have found a remarkable influence of the interference effects on the transport properties, whenever the source-drain voltage is close to the energy of the sideattached quantum dot. Finally, we have given some qualitative insight to the interplay between correlation effects and quantum interference in presence of the proximity induced energy gap.

\section{Acknowledgments}

This work is partly supported by the Polish Ministry of Science and Education under the grant NN202 263138.

\section{References}

[1] R.S. Deacon, Y. Tanaka, A. Oiwa, R. Sakano, K. Yoshida, K. Shibata, K. Hirakawa, S. Tarucha, Phys. Rev. Lett. 104076805 (2010); R.S. Deacon, Y. Tanaka, A. Oiwa, R. Sakano, K. Yoshida, K. Shibata, K. Hirakawa, S. Tarucha, Phys. Rev. B $\mathbf{8 1}$ 121308(R) (2010).

[2] T. Domański, A. Donabidowicz, Phys. Rev. B $\mathbf{7 8}$ 073105 (2008); T. Domański, A. Donabidowicz, K.I. Wysokiński, Phys. Rev. B 76, 104514 (2007).

[3] M. Krawiec, K.I. Wysokiński, Supercond. Sci. Technol. 17, 103 (2004).

[4] Y. Tanaka, N. Kawakami, A. Oguri, Phys. Rev. B $\mathbf{7 8}$ 035444 (2008).

[5] Y. Tanaka, N. Kawakami, A. Oguri, J. Phys. Soc. Jpn. 76, 074701 (2007).

[6] A. Martin-Rodero, A. Levy-Yeyati, Adv. Phys. 60, 899 (2011).

[7] R. Žitko, Phys. Rev. B 81115316 (2010).

[8] J. Barański, T. Domański, Phys. Rev. B 84, 195424 (2011).

[9] H. Haug, A.P. Jauho, Quantum Kinetics in Transport and Optics of Semiconductors, Springer Verlag, Berlin 1996. 